\title{
RESUMEN
}

La invención de la imprenta por Gutemberg a comienzos de la edad moderna iniciló una nueva época y proporcionó a la Reforma, mediante la palabra impresa, un arma eficaz. Impuso una nueva política publicística. La Reforma dispuso de una amplia publicidad. Pronto surgieron, especialmente entre los jesuitas, pioneros de la labor publicistica en pro de la recatolización. Hasta la gucrra de los 30 años se intensificó la guerra politico-publić́stica. Sólo a su término consiguió la necesidad eliminar los antagonismos politico-religiosos.

\section{Kirche zwischen Offentlichkeit und Getto: soziologische und publizistische Perspektiven}

\author{
von Giselbert Deussen
}

Nicht erst seit dem Tod von "Publik“ spricht man von einer Sektenmentalität in der Kirche, die proportional mit dem zeitlichen Abstand vom Konzil zunehme, und von einem Marsch ins Getto, der nicht mehr aufzuhalten sei. Vielen Kritikern schien deshalb auch die Einstellung von „Publik“ lediglich das letzte Siegel auf die längst zugeschlagene Türe zu sein, die die Kirche von der Gesellschaft abschirme. Nun, so sagen sie, beginne die Kirche, sich in ihrem Getto häuslich einzurichten. Man spreche zwar nicht von "Getto", vielmehr zimmere man eine "Theologie der kleinen Herde" und des "heiligen Restes" zusammen, die man aber nach dem Muster einer Sekte konzipiere, die sich nicht für das Ganze der Gesellschaft interessiere und die universale Sendung des Evangeliums verrate. Man fordere eine uniforme Theologie, einheitliche Verhaltensmuster, strengste Observanz und eine straffe Hierarchie. Man restauriere den vorkonziliaren Stil kirchlichen Lebens und stütze sich wieder mit Vorliebe auf kleinbürgerliche und ländliche Gesellschaftsgruppen.

Widersprechen aber solchen Äußerungen nicht jene kirchlichen Aktivitäten, die die Errungenschaften säkularer Kommunikations- und Organisationstechniken auf die Kirche ïbertragen? Sind Synoden, Konferenzen, Räte und schließlich - im publizistischen Bereich - die Bereitstellung von Geldern für ein Gesamtkonzept kirchlicher Publizistik nicht doch Anzeichen eines neuen Aufbruchs der Kirche zu einer gesamtgesellschaftlich relevanten und offenen Interessensgruppe, die sich auf dem Konkurrenzmarkt der Meinungen durchaus verständlich machen und auch durchsetzen kann?

Für und wider einen Marsch der Kirche ins Getto ließen sich viele Stimmen anführen. Hier sollen nur einige Erscheinungen im Informations- und Kommuni-

Dr. Giselbert Deussen ist regelmäßiger Mitarbeiter von CS. Nach dem Studium der Theologie, Sozialwissenschaft und Publizistik promovierte er mit einer Arbeit über die „Ethik der Massenkommunikation bei Papst Paul VI.“. Er ist Pressereferent der Konrad-AdenauerStiftung, Bonn. 
kationsstil der Kirche nach innen und außen betrachtet werden, die vielleicht Indikatoren für entstehende oder entstandene Gettomentalität sein könnten.

\section{Sektenhafte Strukturen in der kirchlichen Publizistik}

Wenn man die heutigen Kommunikationsstrukturen und den von diesen bedingten Umgang mit den Mitteln der Publizistik betrachtet, so schieben sich doch schon einige Merkmale von sektenhaftem Verhalten in den Vordergrund. Als ein Beispiel, dem viele angefügt werden könnten, führe ich das Konzept eines Bischofs von kirchlicher Publizistik an, das dieser in der ersten Ausgabe seiner im neuen Layout erscheinenden Kirchenzeitung zur Aufgabe macht:

„Kirchenzeitung bleib eine Kirchenzeitung! Bringe die Stimme der Kirche! Was Politiker, Parteileute, Verbandsbosse zu verkünden haben, bekommen unsere Leute hinreichend anderswo zu hören. Sage $\mathrm{Du}$, was die Kirche $\mathrm{zu}$ sagen hat - auch Fragen zur Politik, der Wirtschaft, der Schule, der Familie, mit anderen Worten, zu allen Lebensbereichen, besonders aber zu Fragen des Glaubens und des kirchlichen Lebens. Sage es nicht im heute üblichen Pluralismus zur Auswahl, sondern eindeutig und klar, damit die Leute wissen, woran sie sind. Sie sollen durch das Geschriebene nicht verwirrt, verunsichert und schockiert werden. Sie erwarten vielmehr Hilfe, Klärung und vielfach auch entscheidende Weisung.

Liebe Kirchenzeitung, hab nicht den Ehrgeiz, um jeden Preis, modern' oder ,weltoffen' oder ,zügig' zu sein. Sonst müßtest $\mathrm{Du}$ in jeder zweiten Nummer eine Spitze gegen den Heiligen Vater, eine abwertende Bemerkung über Rom, ein Skandälchen aus der Kirchengeschichte oder eine Randbemerkung über, die da oben in Hildesheim' bringen.

Bleib eine Kirchenzeitung! Das heißt für mich: Wende Dich — darf ich es so sagen ans Kirchenvolk. Hab nicht zuerst - wohl auch, aber nicht zuerst - die ,Randchristen' oder ,Nicht-mehr-Christen' im Blick. Das Kirchenvolk sind Deine Leser. Die fordern Klärung, Stärkung und gute Kost; sie soll nicht ohne Salz und Würze sein! Aber es muß die gesunde Lehre bleiben in der Sprache unserer Zeit. Glaubensstärkung brauchen wir, Glaubensunterweisung, Glaubensvertiefung, nicht nur kritisches Hinterfragen ... ${ }^{\boldsymbol{\alpha}_{1}}$

Bei aller Behutsamkeit, mit der solch eine Außerung interpretiert werden muß, kann man doch auf einige Merkmale von Getto- bzw. (soziologisch gesprochen) Sektenmentalität hinweisen. Sind nicht leider — gerade katholische Publizisten beklagen es immer wieder - folgende Verhaltensweisen zunehmend typisch für kirchliches Kommunikationsverhalten geworden?:

1. Angst vor Unruhe, trotz verbaler Bejahung des Pluralismus.

2. Sehnsucht nach Klarheit und Geschlossenheit.

3. Klage über Gegenwart - Sehnsucht nach Vergangenheit.

4. Ständiger Aufruf zum Widerstand gegen den Ungeist und Trend der Zeit (Kulturkritik).

5. Die zunehmende Bedeutungslosigkeit der Kirche wird kirchenpolitisch als "Gesundschrumpfung" und theologisch als das "Schicksal der kleinen Herde“, der "Elite Gottes" gerechtfertigt. 
6. Einschränkung der pastoralen Tätigkeit auf eigene Gruppen-Mitglieder trotz verbaler Betonung der Universalität der Sendung.

7. Vereinheitlichung der Argumente, Einebnung und schließlich Eliminierung differenzierterer Einsichten. Formulierung einer uniformen „Gruppenmeinung “, die dann in intoleranter und intransigenter Weise nach außen durchzusetzen versucht wird.

8. Pauschale Diffamierung von Kritik.

9. Achtung abweichender Meinungen und moralisierende Verdächtigung differenzierender und differierender Argumente.

10. Informationsrestriktion und -selektion.

Diese Symptome zunchmender Gettomentalität in der Kirche verweisen zurück auf eine allen gemeinsame Ursache, auf einen Krankheitsherd, den man - wollte man schwarz sehen - mit an Intensität zunehmender Kommunikationsschwierigkeit, mit "galoppierender Kommunikationsunfähigkeit" der Kirche umschreiben könnte. Besonders deutlich treten diese Symptome augenblicklich an den "Nahtstellen“, auf den "Randpositionen“ des Sozialkörpers Kirche auf, die als Kontakter mit „out-groups“, mit der „Offentlichkeit“, mit der "Welt", fungieren. Unter ihnen sind an erster Stelle die katholischen Publizisten in den publizistischen Institutionen gemeint.

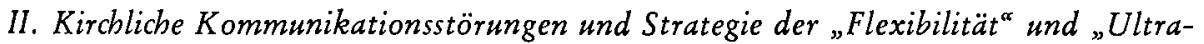 stabilität"}

Bezüglich der Frage, ob die gegenwärtigen Kommunikationsstörungen die Kirche ins Getto führen werden oder schon geführt haben, wird ein Theologe ganz andere Ansichten und Argumente vorbringen als ein Soziologe, ein Bischof andere als sein Kaplan. Wenn im folgenden nur organisations- und kommunikationssoziologisch argumentiert wird, so soll nicht über das Ganze des Phänomens Kirche geurteilt, erst recht aber kein Urteil über die Kirche als Heilsanstalt gefällt werden, sondern eben nur von einem Teilaspekt her.

Die Organisationssoziologie würde die Kirche als ein hochgradig formal-organisiertes System bezeichnen. Nun stehen Systeme und ihre einzelnen Organisationen mit ihrer Umwelt in einem ständigen Kommunikationszusammenhang. Ohne diese Umwelt gäbe es für Systeme keine externen Kommunikationsprobleme. ${ }^{2}$ "Solange Kirche und Welt glaubensmäßig weitgehend gleichgeschaltet waren, bildete die Umwelt der Kirche für diese kein ernstes Problem." ${ }^{3}$ Mit der Emanzipation der Welt von der Kirche wurde letztere zur Absetzung, zur Stellungnahme gegen ein Außen, wenn man so will: zur Segregation, zu Sektionen gezwungen. Bezeichnenderweise ging diese Emanzipationsbewegung der Welt Hand in Hand mit der Entwicklung der neuen technischen Massenkommunikationsmittel und neuer Massenkommunikationssysteme.

Die Kirche hatte seit der Erfindung des Buchdrucks ihr altes Kommunikationsmonopol verloren. Der einzelne Bürger konnte nun - von den etablierten Mächten immer unkontrollierbarer - wenigstens prinzipiell mit seinen Ideen und Alternativargumenten vor ein neu sich bildendes Massenpublikum treten. Aus dem monolithischen, starren Universalpublikum des von der Kirche beherrschten Mittelalters entstanden in der Neuzeit die vielen Publica eigenständig und eigenmächtig räsonnierender Staatsbürger. Kirche, Staat und Gesellschaft waren nicht mehr deckungsgleich. Die 
Kirche sah sich einer varianten Umwelt gegenüber, auf deren Variation und deren Geschichte sie zunehmend weniger Einfluß ausübte. Immer mehr mußte sie sich die Handlungsinitiative entreißen lassen. Sie agierte nicht mehr - sie reagierte: dies aber nicht nur nach außen, sondern auch nach innen, und zwar hier meist mit restaurativer und reaktionärer Tendenz. „Die Organisationssoziologie nennt jene Strategie, die Systeme zu ihrer Bestandserhaltung in einer varianten Umwelt entwickeln müssen, ,Flexibilität ${ }^{‘}$ und ,Ultrastabilität'; gemeint ist damit die Anpassung, die Adaption eines offenen Systems an eine sich wandelnde Umwelt durch strukturellen Wandel ohne Identitätsverlust. ${ }^{\alpha 4}$

Die Frage ist nun allerdings, ob die Kirche wirklich ein offenes System in dem eben beschriebenen Prozeß der Emanzipation der Welt von der Kirche, den man mit einem Schlagwort auch „Säkularisierung“ nennt, geblieben ist. Kirchliche Funktionäre behaupten dies zwar immer wieder, desavouieren jedoch ihre Beteuerungen oft durch ein in bezug auf Kommunikationen äußerst zugeknöpftes Verhalten, dem die Beobachtung der Organisationssoziologie widerspricht, nach der ein offenes System eine große Bereitschaft zum Geben und Empfangen von Nachrichten besitzt und verhältnismäßig geringe Kommunikationsschwierigkeiten nach außen hat. Ein offenes System wird von der Umwelt verstanden und befragt, seine Argumente sind begehrt. In welchem Ausmaß dies für die Kirche noch zutrifft, bedarf je und je einer genauen und ehrlichen Prüfung.

Folgender Trend jedoch scheint zu beweisen, daß die zum Bestand des Systems "Kirche" notwendige Strategie der Flexibilität und Ultrastabilität im Scheitern begriffen ist, daß sie sich auf ihre variante Umwelt nicht einzustellen vermag und daß ihr somit eine Getto- bzw. Sektenexistenz bevorsteht. Viele Menschen ziehen aus der Kirche aus, ohne aus ihr auszutreten. Dahinter steht die Alltagserkenntnis, daß alles auch ohne Kirche geht, und zwar ebenso gut wie vorher, nur noch etwas bequemer. Es steht bestimmt nicht eine spektakuläre Kirchenaustrittsbewegung als Manifestation einer klaren Entscheidung gegen die Kirche mit ihrer Verkündigung bevor. Leider, so möchte man sagen. Denn der schleichende Schwund und die geräuschlose Entfernung vieler aus der Generation der 30- bis 50jährigen sind gefährlicher, weil die vielzitierten Gesundschrumpfungsprozesse sich nicht etwa unter dem Vorzeichen antichristlicher Entscheidungen und einer bewußten antikirchlichen Einstellung ereignen (was für eine relative Offenheit des kirchlichen Kommunikationssystems sprechen würde), sondern sie geschehen im Zeichen einer natürlichen Ausblutung ohne dramatische Konflikte und sozusagen geräuschlos. ${ }^{5}$

Angesichts einer solchen Entwicklung wird der Kirche auch eine forcierte Strategie der Flexibilität wenig nützen. Diese Nutzlosigkeit wird dann jenen Gruppen in der Kirche Oberwasser geben, die mit dem Hinweis auf die Erfolglosigkeit der "Flexiblen ${ }^{2}$ und ihrer Anpassungsstrategien wie gebannt auf die Ultrastabilität der Kirche starren. Traditionalismus, Immobilität und Unzeitgemäßheit - theologisch als christliches Ärgernis und eschatologisches Zeichen interpretiert - würden dann zunehmend das Bild der Kirche in der Offentlichkeit prägen.

In dieser Situation ist die Kirche, will sie nicht zur Sekte werden, auf jene Positionen an ihrer Systemgrenze angewiesen, die auf Flexibilität ausgerichtet sind, auf Dolmetscher, die in zweifacher Richtung vermitteln: nach innen und außen. Diese an der Grenze des Systems Kirche Stehenden - unter ihnen besonders die katholischen Publizisten - tragen die Hauptlast gegenwärtiger Kommunikationsprobleme der 
Kirche. Während die höherstehenden Rollenträger, die mit Entscheidungs- und Weisungsbefugnis, mit Befehlsautorität, ausgestattet sind, ziemlich weit von den Systemgrenzen wegstehen - denn jedes System schirmt die Inhaber höherer Positionen weitgehend von der Umwelt $a b$-, erfahren die Positionen auf den Systemgrenzen die Kommunikationsschwierigkeiten der Kirche mit der Umwelt täglich viel unmittelbarer. Diese haben die Umwelt zu beobachten, einfließende Informationen aufzunehmen und für das System zu verarbeiten. „Aber nicht nur Einflüsse von außen sind zu beobachten, aufzunehmen und zu verarbeiten; denn der Prozeß der Kommunikation ist ja keine Einbahnstraße. Das System will auch nach außen, in die Umwelt hinein kommunizieren. Auch hier sind die Positionsinhaber an der Systemgrenze vielfach unentbehrlich, da sie die oft schwerverständliche und spezifische Sprache des Systems erst in die Sprache der Umwelt übersetzen müssen. " ${ }^{*}$

Diese systemtheoretischen Aussagen beschreiben zwar sehr formal, aber auch zutreffend die Kommunikationsproblematik der Kirche. Wenn es dem System Kirche gelingt, sich an ihren Grenzen in Zukunft fähige Dolmetscher und „Grenzgänger“, die nach außen und innen vermitteln, zu erhalten, dann dürfte sie Chancen haben, den Marsch ins Getto nicht antreten zu müssen. Es wird darauf ankommen, welche Unterstützung, welches Vertrauen diesen Grenzpositionen von der Systemmitte her entgegengebracht werden. Es wird ferner entscheidend sein, in welchem Maße das Zentrum bereit ist. Informationen von diesen anzunehmen, sie an ihren Entscheidungen zu beteiligen. Konflikte zwischen "Grenze" und „Mitte" müssen mit einem großen Vorschuß an Vertrauen ausgetragen werden. Die theologisch so bezeichnete "Distanz der Kirche zur Welt" darf nicht zu einer Distanz zu ihren Vermittlern mit der Welt werden. Prinzipiell müssen Mißverständnisse nach dem alten und weisen Grundsatz des Kirchenrechts: „in dubio pro reo “ gelöst werden. Verdächtigungen und Mißtrauen führen ins Getto, nicht aber verschiedene Meinungen und Standpunkte. So scheint mir nicht die Einstellung von "Publik" als solche Zeichen einer Gettomentalität zu sein, denn journalistisch-ökonomische Erwägungen hatten hier durchaus ihre Berechtigung, sondern die im Zuge der Einstellungsmaßnahmen sich zeigende Schwierigkeiten von Kirchenoffiziellen, ihre Handlungsweise einer breiteren Offentlichkeit einsichtig und verständlich zu machen.

\section{Spezifisch kircbliche Kommunikationsprobleme}

Auftrag und Verkündigung der Kirche müssen als eine spezifische Art sozialer Kommunikation verstanden werden. Hierbei ist es sehr wichtig, Kommunikation als ein zweipoliges Geschehen zu betrachten, da es sich bei Kommunikationen nicht nur um Obertragung von Informationen, sondern zugleich um Informationsverarbeitung handelt. ${ }^{\text {T }}$ Kommunikation als reine Anweisung und Kundgabe gibt es nicht: darum genügt es auch nicht, daß kirchliche Sprache unbesehen aus ihrem Verkündigungsarsenal feststehende Formulierungen hinaussagt, in der irrigen Annahme, das Wort Gottes werde sich schon selbstwirksam seinen Weg in die Herzen der Menschen bahnen. Zur Kommunikation gehören wesentlich die Annahme und das Verstehen durch die "Welt" und ihre Menschen, die gerade nicht so ohne weiteres für das Aussagetum der Kirche disponiert sind. Dies gilt auch zunehmend für die Leser der Kirchenpresse!

Es lassen sich nun aber zwei verschiedene Arten von Kommunikationen unterscheiden, die jeweils ganz spezifische und verschiedene Kommunikationsnetze ausbilden: 
1. die instrumentale Kommunikation, die zwar nur reine Sach- und Wissensdaten vermittelt, aber gerade dadurch dem Menschen seine Orientierung in der Umwelt ermöglicht, und

2. die expressive Kommunikation, bei der Vorstellungs-, Wert- und Gefühlsinhalte ausgetauscht und „Haltungen, Normen und Werte bestätigt oder geändert werden".

Nun leuchtet ein, daß für soziale Gruppen wie die Kirche, die auf die Vermittlung von "Heil“, das heißt, von Haltungen, Normen und Werten aus sind, expressive Kommunikationen die eigentlichen und wichtigen sind. Um nun eine „effektive Durchführung" ihrer Sendung sicherzustellen, um „ihre Ziele zu verwirklichen", müssen sie eine intensive Ubermittlung von expressiven Kommunikationen an ihre unteren Mitglieder unterhalten und Kommunikationshindernisse, die entstehen, ausräumen ". ${ }^{9}$ Mehr noch: Über die Grenzpositionen an ihren Systemgrenzen wollen diese Gruppen ihre expressiven Kommunikationen in ihre Umwelt hinein fortsetzen. Aber die unbedingte Voraussetzung für jede Kommunikation ist die "Gemeinsamkeit der Bezugssysteme“, denn: „Wir können den anderen nur dann verstehen, wenn uns das Gefüge und der Zusammenhang von Erfahrungen und Bedeutungen vertraut ist, innerhalb dessen sich das Leben und Erleben des anderen abspielt. Gemeinsame Bezugssysteme sind also dispositionelle Voraussetzungen für jede Art von Fremdverstehen in der Kommunikation. "

Diese gemeinsamen Bezugssysteme entstehen und bestehen aufgrund gesellschaftlicher Absprachen: „Nur aufgrund gesellschaftlicher Übereinkunft können die Verstehensinhalte sinnvoll weitergegeben werden und kann man damit rechnen, daß sie von den anderen, von einem anderen Wir, einer anderen Gruppe ähnlich verstanden werden. ${ }^{\text {} 11}$ Von hier aus begründet sich kommunikationstheoretisch gesehen die Notwendigkeit des Systems Kirche, sich ständig anpassen, immer wieder Absprachen mit

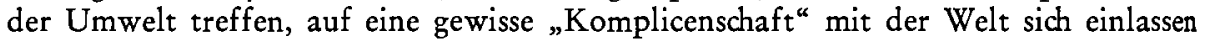
oder - wie Johannes XXIII. es ausdrückte - „Aggiornamento“ üben zu müssen.

Das Problem für auf expressive Weise, also Werte, Normen und Haltungen vermittelnde Kommunikationssysteme wie die Kirche besteht also in folgendem:

Interaktionen und Kommunikationen setzen immer ein gewisses Maß innerer Gleichartigkeit und innerer Ubereinstimmung voraus, eine Kongruenz von Grundüberzeugungen, die den gemeinsamen Bezugsrahmen des Verstehens bilden. ${ }^{12}$ Für die Kirche entsteht so durchaus die Gefahr allzu voreiliger Komplicenschaft mit dem "Zeitgeist", den zu mißachten aber andererseits den Ausbruch aus der kommunizierenden Offentlichkeit, also den Marsch in das Getto bedeuten würde. Ist somit auch die Kirche auf Gedeih und Verderb dem Mechanismus von Angebot und Nachfrage, hier: von Mentalitätsangebot und Mentalitätsnachfrage ausgeliefert? In einem gewissen Sinn ja. Ganz ist sie diesem Mechanismus jedoch ausgeliefert, wenn sie immer nur mißtrauisch reagiert, wenn sie aus Angstlichkeit und mangelnder Zeitgenossenschaft Entwicklungen nicht mehr mitträgt, sondern nur noch griesgrämig beobachtet und verurteilt oder nachträglich auf den Zug der Geschichte aufspringen will, der sie längst überrollt hat.

Am ehesten würde die Kirche noch dem unausweichlich scheinenden Mechanismus entkommen und damit gerade sich selbst treu bleiben, wenn sie nicht bloß reagierte (,Wächteramt"), sondern initiativ agierte, wenn sie, Mißerfolge und Wagnisse nicht scheuend, vorpreschte, den Bezugsrahmen gesellschaftlicher Interaktionen und Kommunikationen durchaus in Rechnung stellend, ihn genau kennend und "barmherzig" 
suf ihn eingehend, ihn gleichwohl immer wieder sprengte, der Erwartung der Menichen vorauseilte, ihre Hoffnungen immer neu weckte und überträfe, ohne es je zu inem Kommunikationsbruch kommen zu lassen.

Gerade dazu aber braucht ein System die "Grenzpositionen“, dazu bedarf es eines sngestörten Informations- und Kommunikationsflusses zwischen dem Zentrum und den Grenzen, dazu bedarf es schließlich auch der horizontalen Kommunikation. Leider bestehen aber ausgerechnet hier gefährliche Kommunikationsschranken ${ }^{13}$, die im operationalen Gefüge der hierarchischen Sozialstruktur der Kirche liegen, in dem die Kommunikation nur von oben nach unten formalisiert ist. Weitere Barrieren entstehen durch die räumliche Trennung der Kommunikatoren, durch die Funktionsüberbelastung der sogenannten Amtsträger, durch die Zentralisierung der Kommunikation, alles in allem: durch den einseitigen Ausbau vertikaler Kommunikationen, durch den einseitigen Informationsfluß überhaupt, durch die undifferenzierte, diffuse Anordnung des Informationsnetzes, durch die mangelnde Ausbildung horizontaler Kontakte und vor allem durch den mangelnden Ausbau eines äußeren Kommunikationsnetzes. ${ }^{14}$

Besonders das im Gefolge der Synode geforderte Gesamtkonzept katholischer Publizistik wird die Kommunikationsprobleme eines Systems mit vorwiegend expressiver und hierarchischer Kommunikation schmerzlich zu spüren bekommen, aber nichtsdestoweniger zu lösen haben. Dabei wird der Mann der Praxis, der katholische Journalist, am ehesten mit der leidigen Tatsache konfrontiert werden, daß sich Kommunikationsschranken sowohl innerhalb wie außerhalb des kirchlichen Systems vor allem aus dem Fehlen eines gemeinsamen Bezugsrahmens des Verstehens ergeben. Hier liegt denn auch für normative soziale Gruppen das eigentliche Problem: „Bei ihren überwiegend expressiven Kommunikationen sind die Schwierigkeiten besonders groß angesichts der in unserer Gesellschaft vorfindlichen Fülle der unverbundenen Wissensformen und der wechselnden Hierarchie von Wissensarten, der Vielfalt von Wortbedeutungen, der Denkmodelle, Abstraktionsstufen und Uberzeugungen religiöser, quasireligiöser und nichtreligiöser Art. ${ }^{15}$ Dabei findet sich der das Getto überwinden wollende Grenzgänger auf einer Fahrt zwischen Skylla und Charybdis: Eine zu vollständige Anpassung an den Umwelt-Bezugsrahmen, ein Aufgehen in ihm, zerstört die Identität und die Sendung der Kirche; doch ein zu großes Absetzen von eben diesem Bezugsrahmen riskiert den Zusammenbruch der Kommunikation mit der Folge der Desintegration und Isolierung. ${ }^{18}$

Das paradoxe Problem lautet: Distanz ohne Distanzierung und Nähe ohne Anbiederung; oder: Distanz ohne Bruch und Nähe ohne Selbstaufgabe. Der Marsch in das Getto würde dann beginnen, wenn die Distanz der Kirche zur Systemumwelt in eine feindliche Haltung umschlagen würde. Die Agression würde dann unter Umständen zur einzig vom System legitimierten Form der Außenkommunikation.

\section{Die Aufgabe der katholiscben Publizistik}

Zur Lösung dieser kirchlichen Kommunikationsprobleme gibt es keine Patentrezepte. Wenn man mich aber auf den Kopf fragen würde nach den Möglichkeiten und der wesentlichen Aufgabenstellung der katholischen Publizistik in diesem Problemsyndrom von Offentlichkeit und Getto, so würde ich auf die beachtliche Zahl von elf Millionen Menschen verweisen, die Käufer von Kirchenpresse sind, die nicht alle 
treue Schafe - gibt es die überhaupt noch? -, die nicht alle Gettochristen sein können, sondern angefochtene, doch mündige Christen von heute sind: vollgesogen mit Welt und doch voll Sehnsucht nach Antworten, die ihnen diese Welt offenbar nicht zu geben vermag (auch nicht die katholische Presse!). Und nun das "Rezept ${ }^{\alpha}$ : Die katholischen Publizisten und ihre Publizistik sollen die Sehnsüchte dieser Menschen zum Sprechen bringen, sollen ihre Anfechtungen und Fragen formulieren und damit ins Gespräch der Kirche und Gesellschaft einbringen. Hier darf es keine von den "Oberhirten" in Kirche und Gesellschaft verordneten Tabus geben.

Hier gilt es zu beherzigen, was Papst Paul VI. einmal katholischen Journalisten anvertraut hat: „Immer mögen Sie Stimme des Volkes sein! Stimme, die es zum Ausdruck bringt; Stimme, die es interpretiert; Stimme, die es formt und hält. Ein moderner Apostel und erfolgreicher Schriftsteller sagt in einem seiner Bücher: ,Ein armer Teufel ist, wer immer nur zuhören muß', das heißt: der, der keine Stimme hat, der sich der Gewalt und der Ubermacht dessen unterwerfen muß, der reden und sich bemerkbar machen kann und dadurch Ubergewicht gewinnt. Seien Sie die Stimme der Armen; seien Sie immer die Stimme des Volkes! “'i7

Anwalt der Armen sein, das heißt für den Publizisten, den Versuch wagen, jene verpönte "schweigende Mehrheit" in der Gesellschaft zum Sprechen bringen, die ihre Meinungen, Interessen und Anliegen nicht artikulieren kann, die „immer nur zuhören muß", die höchsten ihre Meinung dadurch zum Ausdruck bringen kann, daß sie den Kommunikatoren ihre Zustimmung schenkt oder verweigert. Hinhorchen auf den „sprachlosen“ Zeitgenossen, den "kleinen Mann", und nicht immer wieder die Meinung der Offiziellen nachbeten, die nach Möglichkeit auch noch keinem wehe tut, besonders den "Mächtigen" in Kirche und Gesellschaft nicht, - dies wäre ein Programm für eine Publizistik und Kommunikation, die nur sehr schwer in die Gefahr käme, in ein Getto zu kommen, oder Komplice einer Offentlichkeit zu werden, die vorher hinter den Kulissen von den Mächtigen vorgekartet wurde. Sie würde Offentlichkeit schaffen gerade dort, wo sie - als kritische - von den Interessen der kirchlichen und weltlichen Drahtzieher nicht gewünscht würde, aber gleichwohl im Sinne der Selbstbestimmung der „schweigenden Mehrheit“ vonnöten wäre.

Denn bei dem bewegten und bunten Bild, das die demokratische Offentlichkeit einer pluralistischen Gesellschaft oberflächlich bietet, vergißt man sehr leicht, daß es immer nur einigen wenigen Repräsentanten gelingt, vor eine breitere Offentlichkeit zu treten und das große Sagen zu haben. Die moderne Medienkommunikation wird - wie alle menschlichen Beziehungen - immer komplexer, komplizierter und undurchschaubarer; sie kann zunehmend nur noch von ausgebildeten und hochqualifizierten Spezialisten wahrgenommen werden. Wenn diese nun den Kontakt mit der „schweigenden Mehrheit " verlieren, droht das Wort von der "Demokratisierung " zu einer Verhöhnung der Massen zu werden: „Ein armer Teufel ist, wer immer nur zuhören muß." Wenn man ein Schlagwort aus der Kapitalismuskritik der sogenannten Neuen Linken aufgreifen will, so muß man feststellen, daß den modernen Demokratien aufgrund ihrer Offentlichkeitsstruktur und der Gesetzmäßigkeiten der Herstellung von Offentlichkeit ein "Medienfaschismus“, eine Diktatur der Medieneigner sowohl wie der Medienspezialisten, droht. Die Kommerzialisierung und Technokratisierung der Offentlichkeit bedroht die Kommunikation, wenn diese nur das Privileg einiger weniger ist. Es bietet sich ein Schauspiel, das nur einige Akteure, im übrigen aber nur schweigende Zuschauer kennt. 
Deshalb steht dem Publizisten eine geistige Disziplin wohl an, die die „Stimme des Volkes" nicht vorschnell als "Massengeschmack", "falsches Bewußtsein", als unter seinem Niveau stehend abtut, sondern diese Stimme einbringt in ein wirklich demokratisch geführtes Gespräch in Kirche und Gesellschaft. "Wenn die Mehrheit nicht zum Zuge kommt, weil sie nicht spricht, kann von Demokratie nicht die Rede sein ... $\mathrm{Ob}$ es jemand ehrlich meint mit der Forderung, Demokratie in der Kirche zu verwirklichen ..., wird man daran erkennen, ob er sich bemüht, die ,schweigende Mehrheit ${ }^{*}$ zu Wort kommen zu lassen, selbst auf die Gefahr hin, daß sie, sobald sie spricht, gerade ihm energisch widerspricht. " 18

Das Sprachlose und den Sprachlosen zum Sprechen bringen - nach innen und nach außen - das scheint mir eine spezifisch christliche Aufgabe in der heutigen Zeit zu sein. Die katholische Presse und Publizistik in ihrer "Grenzgängerpostion " wäre eigentlich prädestiniert dazu. Sie kann eine Schlüsselfunktion erfüllen in der Wahl der Kirche zwischen Offentlichkeit und Getto.

\section{Anmerkungen:}

1. Dieses Zitat entnehme ich den "Dokumenten" rom 16. 10. 1972 der Bischöflichen Pressestelle Hildesheim, die sich ausschließlich mit der Zurückweisung des von mir gewagten Unterfangens befaßte, diese Außerung des Bischofs von Hildesheim in einem Vortrag bei der Jahresversammlung der Arbeitgemeinschaft Katholische Presse am 4. 10. 1972 in Münster als Beispiel für ein Denken in Getto-Kategorien angeführt zu haben. Diese Dokumentation, die weitere Zitate zum Zwecke der Dokumentation des Gegenteils anführt, empfehle ich als Fundgrube für weitere Belege der von mir aufgestellten Behauptungen.

2. Vgl. N. Luhmann: Sozialogie als Theorie sozialer Systeme, in: „Kölner Zeitschrift für Soziologie und Sozialpsychologie" 19:1967, S. 615-644.

3. W. Weber: Probleme der Kirche als Informations- und Kommunikationssystem, in: A. Exeler (Hrsg.): Fragen der Kirche heute, Würzburg 1971, S. 148.

4. Ebenda.

5. G. Jacob: Die Kirche im Jahre 1985, in: „Der Spiegel“ 1968.

6. W. Weber: a.a.O., S. 151.

7. Vgl. N. Luhmann: Funktionen und Folgen formaler Organisation, Berlin 1964, S. 192.

8. A. Etzioni: A Comparative Analysis of Complex Organisations on Power, Involvement, and their Correlates. Third Printing, New York 1965, S. 138.

9. Ebenda.

10. Ph. Lersch: Der Mensch als soziales Wesen, München 1964, S. 66 und 69.

11. G. Bormann: Kommunikationsprobleme in der Kirche, in: J. Matthes: Kirche und Gesellschaft. Einführung in die Religionssoziologie II, Hamburg 1969, S. $169 \mathrm{f}$.

12. Vgl. Henk Prakke, Kommunikation der Gesellschaft, Münster 1968, S. 140.

13. Vgl. A. Etzioni: a.a.O., S. 139.

14. Vgl. G. Bormann: a.a.O., S. 170 f.

15. Ders.: a.a.O., S. 171; vgl. G. Gurvitch: Wissenssoziologie, in: Die Lehre von der Gesellschaft, hrsg. von G. Eisermann, Stuttgart 1958, S. $436 \mathrm{ff}$., $432 \mathrm{ff}$.

16. Vgl. G. Bormann: a.a.O., S. 172.

17. Paul VI.: Ansprache vom 20. 9. 1969 an die Mitarbeiter der Wochenzeitung "La Voce del Popolo" (Brescia); hier zitiert nach: G. Deussen: Ethik der Massenkommunikation bei Papst Paul VI., Paderborn 1973, S. 193.

18. O. B. Roegele: Zum Streit um das Bild des Priesters in der Gegenwart, in: „Lebendige Seelsorge“ $21: 1970$, Heft 1/2, S. 3. 
The author fears that the church nowadays, in conflict between publicity and Ghetto - based on today's critics - is tending more to the latter, although it will not dominate, if they do not agree in granting an appropriate liberty to religious journalism. Press and journalism in their functions as border crossers are predestinated to have a key position in the choice of the church between publicity and Ghetto. The paradoxical problem with regard to the relation of the church with publicity and the world is "Distance without detachment" and "nearness without attachment" or "distance without break" and "nearness without subjection". The way into the Ghetto starts "once the distance of the church to the system environment turns into an adverse attitude".

\section{RESUME}

L'auteur craint que l'Eglise, dans ses démélés avec le temps présent entre public et guetto mesuré aux critères actuels - ne tende à nouveau toujours plus fortement vers ce dernier, pour le moins ne se rende que difficilement maître de ce danger si elle ne concède pas à la publicistique spirituelle l'espace libre dont elle a besoin. La presse et la publicistique sont, dans leur "position en marge“, prédestinées à remplir une fonction-clé dans le choix de l'Eglise entre le public et le guetto. Le problème paradoxal pour le rapport de l'Eglise face au public et face au monde est le suivant: „Distance sans désolidarisation et proximité sans familiarités; ou bien: Distance sans rupture et proximité sans abandon de soi“. La marche vers le guetto commencera "quand la distance de l'Eglise par rapport à cet environnement de systèmes se sera retournée en attitude hostile“.

\section{RESUMEN}

El auor teme que la Iglesia, en su contraposición al mundo actual y ante la alternativa de elegir entre vida pública y getto - a tenor de los criterios actuales - se incline de nuevo progresivamente hacia esta última eventualidad, que será solo un mal menor si no se deja al publicismo eclesial adecuado margen de libertad de acción. Prensa y publicismo están predestinados a cumplir una función clave en la elección de la Iglesia entre vida pública y getto. Puede formularse así el paradógico dilema en las relaciones de la Iglesia con la vida pública y con el mundo: „Distancia sin distanciamiento y proximidad sin compadreo o distancia sin ruptura y proximidad sin propio renunciamiento “. La marche hacia el getto comienza "cuando la distancia de la Iglesia respecto del sistema mundano circundante se environment turns into an adverse attitude". 